\title{
Theoretical Calculations and Modeling for the Molecular Polarization of Furan and Thiophene under the Action of an Electric Field Using Quantum Similarity
}

\author{
Alejandro Morales-Bayuelo ${ }^{1,2}$ and Ricardo Vivas-Reyes ${ }^{1}$ \\ ${ }^{1}$ Grupo de Química Cuántica y Teórica, Facultad de Ciencias Exactas y Naturales, Universidad de Cartagena, \\ Programa de Química, 130015 Cartagena de Indias, Colombia \\ ${ }^{2}$ Departamento de Ciencias Químicas, Universidad Nacional Andres Bello, Republica 275, 8370146 Santiago, Chile
}

Correspondence should be addressed to Alejandro Morales-Bayuelo; alejandromb5@hotmail.com and Ricardo Vivas-Reyes; rvivasr@unicartagena.edu.co

Received 19 September 2013; Revised 25 November 2013; Accepted 30 November 2013; Published 17 March 2014

Academic Editor: Aijun Du

Copyright (C) 2014 A. Morales-Bayuelo and R. Vivas-Reyes. This is an open access article distributed under the Creative Commons Attribution License, which permits unrestricted use, distribution, and reproduction in any medium, provided the original work is properly cited.

\begin{abstract}
A theoretical study on the molecular polarization of thiophene and furan under the action of an electric field using Local Quantum Similarity Indexes (LQSI) was performed. This model is based on Hirshfeld partitioning of electron density within the framework of Density Functional Theory (DFT). Six local similarity indexes were used: overlap, overlap-interaction, coulomb, coulombinteraction, Euclidian distances of overlap, and Euclidean distances of coulomb. In addition Topo-Geometrical Superposition Algorithm (TGSA) was used as a method of alignment. This method provides a straightforward procedure to solve the problem of molecular relative orientation. It provides a tool to evaluate molecular quantum similarity, enabling the study of structural systems, which differ in only one atom such as thiophene and furan (point group $\mathrm{C}_{2 \mathrm{v}}$ ) and cyclopentadienyl molecule (point group $\mathrm{D}_{5 \mathrm{~h}}$ ). Additionally, this model can contribute to the interpretation of chemical bonds, and molecular interactions in the framework of the solvent effect theory.
\end{abstract}

\section{Introduction}

In chemistry the concept of molecular polarization is a key concept often used to rationalize a lot of molecular properties on the basis of their constituent atoms, geometry, or functional groups. Polarizability allows us to make qualitative judgements about structure and translate them into first approximation of expected properties and chemical reactivity. We can expect, for example, that electrophiles to attack positions with relatively high electron density and polarizability. Nucleophiles should attack relatively low electron density and polarizability. Thus a lot of properties such as boiled point, solubility, melting point, softness, and molecular reactivity depend on its polarizability properties.

In this study we related quantum molecular similarity to the molecular polarity, in order to find a theoretical model. Thiophene and furan molecules (point group $\mathrm{C}_{2 \mathrm{v}}$ ) and cyclopentadienyl molecule (point group $\mathrm{D}_{5 \mathrm{~h}}$ ) were taken as model of this application. In this contribution a local similarity index based on the Hirshfeld partitioning technique was used [1]. This approach provides a tool to evaluate molecular quantum similarity, enabling the study of structural systems, which differ in only one atom.

One of the carbon-carbon $(\mathrm{C}-\mathrm{C})$ bonds of the cyclopentadienyl and $(\mathrm{C}-\mathrm{S})$ bond from thiophene and $(\mathrm{C}-\mathrm{O})$ bond of furan were taken as molecular fragments; these three fragments $(\mathrm{C}-\mathrm{S}, \mathrm{C}-\mathrm{O}$, and $\mathrm{C}-\mathrm{C})$ were approximated as diatomic molecule. Taking into account this idea which proposed a theoretical model allows us to relate the molecular polarity under the action of an electric field with a series of quantum similarity descriptors [2-12].

This study is in line with the increasing importance of applying quantum mechanically based techniques to study the properties and reactions of molecules [2, 3]. Quantum 
chemically based indexes are receiving increasing attention; for example, Molecular Quantum Similarity Index (MQSI) is based on the electron density proposed by Carbó-Dorca et al. [2-12] almost three decades ago and is the prototype of such an index that continues to play an important role.

\section{Theory and Computational Details}

2.1. Molecular Polarization of Molecules in an External Electric Field. To achieve a characterization of the molecular polarization was used the molecular model of polarization of two atoms identify from the point of view of its quantum states proposed by Khvalchenko [13]. Using this idea in this work, the $\mathrm{C}^{1}-\mathrm{C}^{2}$ bond of the cyclopentadienyl molecule was taken as model the polarization of two identical atoms under the action of an electric field, for a transition $2 s \rightarrow 3$ p orbital.

In this model cyclopentadienyl was designated as molecule A. Suppose that each carbon atom is in the $2 \mathrm{~s}$ or $3 \mathrm{p}$ state. All possible initial states of the system under study are described by the wave function depicted in the following:

$$
\Psi_{m}^{L}=\sum_{\mu} s_{L, \mu, m-\mu}^{(l, \bar{l})} \Psi_{\mu} \bar{\Psi}_{m-\mu},
$$

where $s_{L, \mu, m-\mu}^{(l, \bar{l})}$ are the Wigner coefficients [14] and the over barred symbols refer to the states of the second atom. Using the relation (1) determines 16 initial states of the system of atoms (see Appendix A).

2.2. Linearly Polarized Field. Under the action of an electric field, the atoms are behaving as $E=\left(E_{1} i+E_{2} j+E_{3} k\right) \cos (\omega t)$. The quantum states of this system are given by the following:

$$
i \hbar \frac{\partial}{\partial t}=\left(\widehat{\mathbf{H}}_{0}+\widehat{\mathbf{H}}_{0}+\widehat{\mathbf{V}}\right) \Psi \text {. }
$$

To this equation we have

$$
\Psi=\sum_{k=1}^{16} a_{k} \Psi_{k} \exp \left(-i \omega_{k} t\right)
$$

Taking into account that

$$
\omega_{k}= \begin{cases}2 \omega_{1}, & k=1 \\ \omega_{1}+\omega_{2}, & k=\overline{2,7} \\ 2 \omega_{2}, & k=\overline{8,16},\end{cases}
$$

$\hbar \omega_{1}$ and $\hbar \omega_{2}$ are the energies of the $2 \mathrm{~s}$ and $3 \mathrm{p}$ and the respective Hamiltonians $\widehat{\mathbf{H}}_{\mathbf{0}}$ and $\widehat{\mathbf{H}}_{\mathbf{0}}$ of the atoms and $\widehat{\mathbf{V}}=-(\mathbf{D}, \mathbf{E})$, $\mathbf{D}=\mathbf{d}+\overline{\mathbf{d}}, \mathbf{d}+\overline{\mathbf{d}}$ are the operators of the dipole moments of the atoms; from (2) it is possible to obtain the following differential system $(\dot{a})$ :

$$
i \hbar \dot{a}=V a \text {. }
$$

To simplify (5), was used the method of successive approximations given in (5), in this way was obtained a set of coefficients as first approximation:

$$
a=I-\int_{0}^{t} V(\tau) d \tau
$$

where $(a)$ is a square matrix of order 16 and the columns are the functions $\left|\Psi_{\mathrm{k}}\right\rangle$ of the atoms-plus-field system in the initial states given (see Appendix A). For calculation, the polarization of this system was used (7), for the molecule A.

Consider the following:

$$
P_{\mathrm{kA}}=S p \widehat{\rho}_{\mathrm{k}} \mathbf{D}
$$

where $\hat{\rho}_{\mathrm{k}}=\left|\Psi_{\mathrm{k}}\right\rangle\left\langle\Psi_{\mathrm{k}}\right|$ and $\mathrm{D}$ is the operator constructed using as basis the functions of (3). This allows us to obtain the expressions for the corresponding initial states given by (2) (see Appendix B).

In this study, it was proposed that the multiplication of (7) by the similarity descriptors gives a new local descriptor that we call "Induced Polarity Molecular Descriptor (IPMD)" and it is postulated as quantifier of (dis)similarity between the molecular polarization and the quantum similarity using density function coordinates matrix (see Appendix C).

2.3. Similarity Indexes. Carbó-Dorca et al. [2, 3, 10, 11, 15, 16] defined the Euclidean distance, $D_{\mathrm{AB}}$, between the electron densities $\rho_{\mathrm{A}}(r)$ and $\rho_{\mathrm{B}}(r)$ of two molecules $\mathrm{A}$ and $\mathrm{B}$ as follows:

$$
\begin{aligned}
D_{\mathrm{AB}}= & \int\left|\rho_{\mathrm{A}}(r)-\rho_{\mathrm{B}}(r)\right|^{2} d r=\int \rho_{\mathrm{A}}^{2}(r) d r \\
& +\int \rho_{\mathrm{B}}^{2}(r) d r-2 \int \rho_{\mathrm{A}}(r) \rho_{\mathrm{B}}(r) d r .
\end{aligned}
$$

In the MQSI framework the axiomatic properties of the Euclidean distance can be mentioned and defined according to M. M. Deza and E. Deza [11] as follows.

Let $\mathbf{H}$ be a set. A function $\mathrm{G}: \mathbf{H} \times \mathbf{H} \rightarrow \mathbf{R}^{+}$is called a distance (or dissimilarity) on $\mathbf{H}$. If and only if for all $\rho_{\mathrm{A}}(r), \rho_{\mathrm{B}}(r) \in \mathbf{H}$, there holds the following:

(i) $D\left(\rho_{\mathrm{A}}(r), \rho_{\mathrm{B}}(r)\right) \geq 0$ (nonnegativity)

(ii) $D\left(\rho_{\mathrm{A}}(r), \rho_{\mathrm{B}}(r)\right)=D\left(\rho_{\mathrm{B}}(r), \rho_{\mathrm{A}}(r)\right)$ (symmetry)

(iii) $D\left(\rho_{\mathrm{A}}(r), \rho_{\mathrm{B}}(r)\right)=0$ (reflexivity).

Use of these mathematical properties and axiomatic in this study explained the concept of (dis)similarity in quantum object sets supported in a pre-Hilbert vector space, where the scalar product and an attached norm can be defined [15]. This index can be represented geometrically using the concept of cosine of the angle subtended between the functions of densities, considering them as vectors; this index is defined mathematically as follows:

$$
R_{\mathrm{AB}}=\frac{\int \rho_{\mathrm{A}}(r) \rho_{\mathrm{B}}(r) d r}{\left[\left(\int \rho_{\mathrm{A}}^{2}(r) d r\right)\left(\int \rho_{\mathrm{B}}^{2}(r) d r\right)\right]^{1 / 2}}=\frac{Z_{\mathrm{AB}}}{\sqrt{Z_{\mathrm{AA}} Z_{\mathrm{BB}}}} .
$$

Equation (9) involves the overlap integral $Z_{\mathrm{AB}}$, often called Molecular Quantum Similarity Measure (MQSM), between the electron densities of molecules $\mathrm{A}$ and B. $Z_{\mathrm{AA}}$ and $Z_{\mathrm{BB}}$ are called the Molecular Quantum Self-Similarity Measures of molecules A and B [17, 18] (see Appendix C). 
2.3.1. Local Quantum Similarity Index (LQSI) for the Structures. To obtain the molecular quantum similarity from the point of view of atoms in this study the Hirshfeld partitioning technique was used [1], which suggested that the electron density of a molecule can be distributed in contributions at atomic level. This approximation is widely used in studies ranging from the prediction of atomic properties to local chemical reactivity [19]. In this work was used for the conversion of the global Carbó index (9) into a local index; the calculation of LQSI with DFT is a very used method [19-23].

Starting from Hirshfeld idea that the atomic contributions are proportional to the weight $\left(w_{\mathrm{S}}(r)\right)$ of the electronic density of the molecule isolated in the so-called promolecular density [1], local contributions in electronic density can be considered as the summations from the local perspective obtaining atoms-in-molecules densities by defining the "stock-amount" or weight of a sulphur atom (S) in the electron density at $r$ that can be written as follows:

$$
w_{\mathrm{S}}(r)=\frac{\rho_{\mathrm{S}}^{0}(r)}{\sum_{X} \rho_{X, \mathrm{~S}}^{0}(r)} .
$$

Here, $\rho_{\mathrm{S}}^{0}(r)$ is the electron density of the isolated sulphur atom in the molecule, and $\sum_{X} \rho_{X, S}^{0}(r)$ is the promolecular density of the molecule, obtained as the sum of the isolated atom densities in the same molecular geometry as the actual molecule. The Hirshfeld atomic electron density of the atom a in the molecule can be calculated as follows:

$$
\rho_{\mathrm{S}}(r)=w_{\mathrm{S}}(r) \rho_{\mathrm{S}}(r) .
$$

Analogously, for the cyclopentadienyl

$$
w_{\mathrm{C}}(r)=\frac{\rho_{\mathrm{C}}^{0}(r)}{\sum_{Y} \rho_{Y, \mathrm{C}}^{0}(r)} .
$$

The numerator of the Carbó index then becomes

$$
Z^{\text {local }, \mathrm{S}}=\int w_{\mathrm{S}} \rho(r) d r=\int\left(\frac{\rho_{\mathrm{S}}^{0}(r)}{\sum_{X} \rho_{X, \mathrm{~S}}^{0}(r)}\right) \rho_{\mathrm{S}}(r) d r .
$$

And, for the cyclopentadienyl,

$$
Z^{\text {local }, \mathrm{C}}=\int w_{\mathrm{C}} \rho(r) d r=\int\left(\frac{\rho_{\mathrm{C}}^{0}(r)}{\sum_{\mathrm{X}} \rho_{\mathrm{X}, \mathrm{C}}^{0}(r)}\right) \rho_{\mathrm{C}}(r) d r,
$$

whereas the self-similarities $Z_{\mathrm{SS}}=\left(Z^{\text {local,S }}\right)^{2}$ and $Z_{\mathrm{CC}}=$ $\left(Z^{\text {local, },}\right)^{2}$ can be written analogously in terms of atomic contributions, using (13) and (14) in the expression of the Carbó index (9); the global index is converted in a local analogue:

$$
\begin{aligned}
& R_{\mathrm{SC}}^{\text {local }} \\
& =\frac{Z^{\text {local }, \mathrm{S}} Z^{\text {local,C }}}{\sqrt{\left(Z^{\text {local }, \mathrm{S}}\right)^{2}\left(Z^{\text {local }, \mathrm{C}}\right)^{2}}}
\end{aligned}
$$

$$
\begin{gathered}
=\left(\int\left(\frac{\rho_{\mathrm{S}}^{0}(r)}{\sum_{X} \rho_{X, \mathrm{~S}}^{0}(r)}\right)\left(\frac{\rho_{\mathrm{C}}^{0}(r)}{\sum_{Y} \rho_{Y, \mathrm{C}}^{0}(r)}\right) \rho_{\mathrm{S}}(r) \rho_{\mathrm{C}}(r) d r\right) \\
\times\left(\left[\int\left(\frac{\rho_{\mathrm{S}}^{0}(r)}{\sum_{X} \rho_{X, \mathrm{~S}}^{0}(r)}\right)^{2} \rho_{\mathrm{S}}(r) \rho_{\mathrm{S}}(r) d r\right]^{1 / 2}\right. \\
\left.\times\left[\int\left(\frac{\rho_{\mathrm{C}}^{0}(r)}{\sum_{Y} \rho_{Y, \mathrm{C}}^{0}(r)}\right)^{2} \rho_{\mathrm{C}}(r) \rho_{\mathrm{C}}(r) d r\right]^{1 / 2}\right)^{-1},
\end{gathered}
$$

where the global index is partitioned in atomic contributions, given as result considerations at local level.

2.4. Alignment Structures and Computational Details. In the quantum similarity field the Carbó and Hodgkin-Richards indexes are the most used $[2,3,24]$. These indexes are dependent on the relative orientation of the study structures. Taking into account that this dependence has proposed many alignment methodologies, ranging from those used in the CoMFA and CoMSIA methods $[25,26]$ to the Topo-Geometrical superposition algorithm (TGSA) [23] which is based on comparison of the types of atoms, distances between them were used in this work. The Topo-Geometrical Superposition Algorithm (TGSA) proposed by Girones et al. [23] begins with the recognition of the largest common substructure in the aligned molecules and this type of search algorithms constitutes an important field of scientific interest [27].

All molecules were optimized using B3LYP/6-31+G(d) [28-30] in the Gaussian 09 program [31]. The molecules properties such as bond distances, dipole moments, and atomic charges were calculated using ChemCraft 1.6 program [32].

\section{Results and Discussion}

The calculations of local similarity are well supported in DFT. One of the central postulates of quantum mechanics is that if the wave function of the system is known, every socalled observable property for an $\mathrm{N}$-electron molecule may be obtained by straightforward integration of

$$
\begin{aligned}
& \Omega[\Psi] \\
& \quad \equiv \iint \ldots \int \Psi^{*}\left(x_{1}, \ldots, x_{N}\right) \hat{\Omega} \Psi\left(x_{1}, \ldots, x_{N}\right) d x_{1} \cdots d x_{N},
\end{aligned}
$$

where $(\Omega)$ is the observable property of the system. This is located where the electron density is higher in a molecule. It is found that the electronic density values are higher in the volumes defined by the subvalence regions of each atom. It is also well known that in those regions, chemical bonding produces only small changes in the electronic density, in comparison with isolated atoms. This suggests an approximated electron density that we can write as the sum of the isolated atomic densities [19-23].

In this model the induced molecular polarization by electric field on thiophene and furan can be expressed 


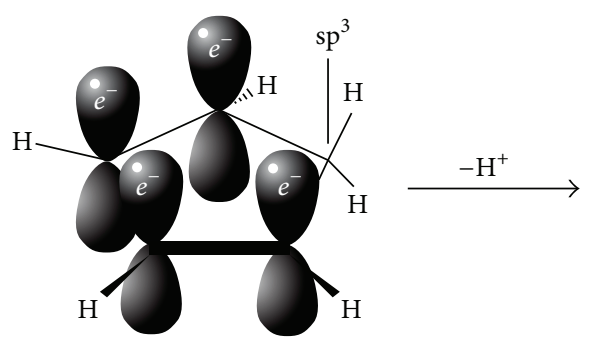

Cyclopentadiene

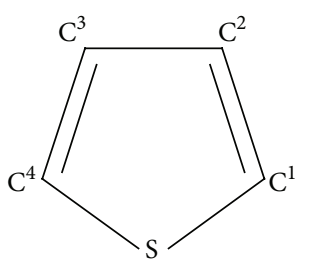

(A)

(a)

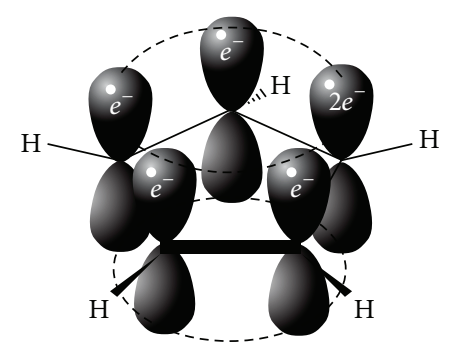

Cyclopentadienyl anion

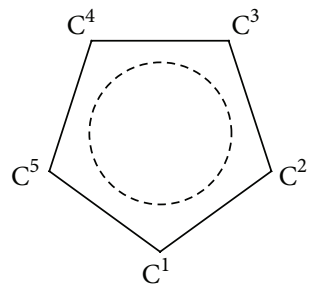

(B)

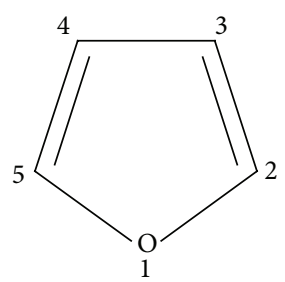

(C)

(b)

Figure 1: (a) Formation of the resonance in cyclopentadienyl molecule (A) thiophene structure (point group $\mathrm{C}_{2 \mathrm{v}}$ ), (B) cyclopentadienyl molecule (point group $\mathrm{D}_{5 \mathrm{~h}}$ ), its atoms used for superposition being labelled, and finally $(C)$ furan structure (point group $\mathrm{C}_{2 \mathrm{v}}$ ).

TABLE 1: Local similarity indexes (numerical and analytical methods) and Euclidean distances, using global densities for thiophene, furan versus cyclopentadienyl.

\begin{tabular}{lcccccc}
\hline Cyclopentadienyl $^{\mathrm{a}}$ & $Z_{\mathrm{CP}}(\mathrm{O})^{\mathrm{b}}$ & $Z_{\mathrm{CP}}(\mathrm{C})^{\mathrm{c}}$ & LSI $(\mathrm{O})^{\mathrm{d}}$ & LSI $(\mathrm{C})^{\mathrm{e}}$ & $D_{\mathrm{CP}}(\mathrm{O})^{\mathrm{f}}$ & $D_{\mathrm{CP}}(\mathrm{C})^{\mathrm{g}}$ \\
\hline Versus thiophene $^{\mathrm{h}}$ & 2.994 & 196.350 & 0.972 & 0.997 & 0.410 & 0.966 \\
Versus furan $^{\mathrm{h}}$ & 3.441 & 211.863 & 0.912 & 0.991 & 0.871 & 2.009 \\
\hline
\end{tabular}

${ }^{a}$ Point group $\mathrm{D}_{5 \mathrm{~h}}$.

${ }^{\mathrm{b}}$ Interaction of overlap-numerical method.

${ }^{\mathrm{c}}$ Interaction of coulomb-numerical method.

${ }^{\mathrm{d}}$ Local similarity index of overlap (see Appendix C).

${ }^{\mathrm{e}}$ Local similarity index of coulomb (see Appendix C).

${ }^{\mathrm{f}}$ Euclidean distance of overlap.

${ }^{g}$ Euclidean distance of coulomb.

${ }^{\mathrm{h}}$ Point group $\mathrm{C}_{2 \mathrm{v}}$.

in terms of the molecular polarization of cyclopentadienyl (Figure 1). Carbó indexes help to relate thiophene and furan with the cyclopentadienyl. These indexes were applied to these two molecules in which $(2 s \rightarrow 3 p)$ orbital transitions occur. These transitions are independent of the dielectric constant of the medium. In this since, in this study is explored the induced polarization in different quantum states under the action of an electric field (see Appendices A and B).

The induced polarizability values for the initial states $\left(P_{\mathrm{kA}}\right)$ are calculated using $(7)$. Thelocal similarity indexescancalculate the structural and electronic similarity between thiophene, furan, and cyclopentadienyl with their respective Euclidean distances. A low value in the Euclidean distance indicates that these compounds are structurally and electronically very similar. In this sense the similarity indexes are shown in Table 1.

In Table 1 the higher overlap index is 0.972 to the thiophene with a coulomb index of 0.997 , while to the furan the overlap index is 0.912 and the coulomb index is 0.991 , giving insights into the steric and electronic effects, structural parameters such as bond length, atomic size, and also orbital differences between the $\mathrm{p}$ orbital of the sulphur and oxygen atom with respect to the $\mathrm{sp}^{2}$ orbital of the carbon atom in cyclopentadienyl (symmetry $\mathrm{D}_{5 \mathrm{~h}}$ ). On the other hand, the electronic effects such as the resonance are more relevant in the thiophene with respect to the furan due to the presence of an additional electron in comparison with cyclopentadienyl. These results were supported by a Euclidean distance of overlap of 0.410 and a Euclidean distance of coulomb 0.966 to the thiophene and a Euclidean distance of overlap of 0.871 and a Euclidean distance of coulomb 2.009.

All carbon atoms in thiophene and furan are $\mathrm{sp}^{2}$ hybridized; thus we have four electrons remaining for pi bonding (see Figure 1). The sulphur and oxygen atoms have two lone pairs and two bonds (which normally lead to $\mathrm{sp}^{3}$ hybridization). In contrast, in a sulphur atom, the electrons of the lone 

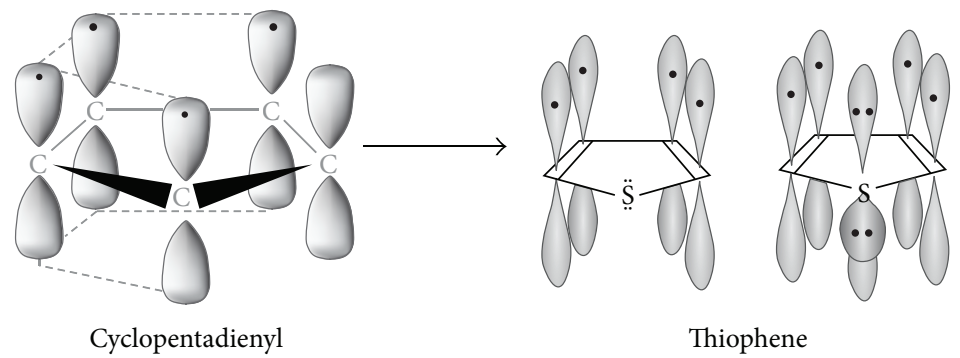

Figure 2: Comparison of the resonant systems of thiophene with respect to the cyclopentadienyl.

pair are promoted to the $\mathrm{p}$ orbital and the hybridization of all the other carbons is produced (three $\mathrm{sp}^{2}$ and one p); therefore it cannot be considered $(4 n+2)$ electronic system. On the other hand, in Figure 2, we can see that the electronic pair over the negatively charged carbon of the cyclopentadienyl anion obeys the $(4 n+2)$ electron rule. The $\mathrm{sp}^{2}$ carbon of the cyclopentadienyl anion (quantified by the similarity indexes with respect to thiophene ring) shows a strong acidic character. These particular facts generate the electronic dissimilarity of carbon with respect to sulphur in thiophene ring.

If all the carbons are $\mathrm{sp}^{2}$ hybridized then we have four electrons remaining for pi bonding. Sulphur atom has two lone pairs and two bonds (which would normally make four $\mathrm{sp}^{3}$ hybridized orbitals). If one of sulphur's lone pairs is promoted to a $\mathrm{p}$ orbital it could become hybridized like all the other carbons (three $\mathrm{sp}^{2}$ and one $\mathrm{p}$ ) and as a result there would be $(4 n+2)$ electrons from sulphur atom in the pi bonding. These details generate the electronic (dis)similarity of carbon atom (C) with respect to sulphur (S) in thiophene molecule.

In Figure 3, we can see the contrast of the electronic properties for cyclopentadienyl. These have more anionic characteristic in their behaviour in accordance with the rule of $(4 n+$ 2 electrons) in the thiophene. The sp2 carbon of cyclopentadienyl (quantified by the overlap local index of similarity with respect to the thiophene) is more acidic than we expected (as the alcohol acid character) because the conjugate base, the cyclopentadienyl anion, is aromatic and therefore very stable.

The Euclidean distances of overlap are higher than the corresponding coulomb distances (Table 1). The bond lengths in the structures (Figure 4) and their respective resonant systems (see Figures 1 and 3) have significant differences. This is evidenced by the low value in the local similarity index of overlap with respect to the coulomb index.

In Figure 4, we can see the lengths of the cyclopentadienyl carbon-carbon bonds $(\mathrm{C}-\mathrm{C})$ and thiophene sulphur-carbon (C-S) bonds. The $\mathrm{C}-\mathrm{C}$ bond lengths are equal to $1.445 \AA$. This means that our initial hypothesis to not consider the polarization of atoms near the site of interest is well justified.

The trends of the dipole moment and total atomic charges are displayed in Figure 4. In the case of thiophene the dipole moment is oriented towards the sulphur atom, although it has similar electronegativity to the carbon atom (Pauling electronegativity $\chi(\mathrm{C}): 2.55$ and $\chi(\mathrm{S}): 2.58)$. This small difference causes a decrease of orbital symmetry in

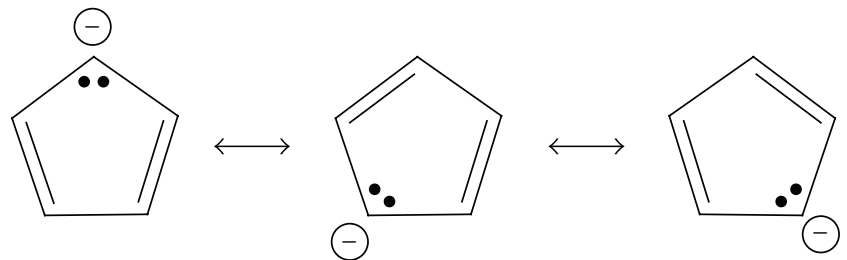

FIGURE 3: The cyclopentadienyl anion resonance.

the sulphur atom. In this case, it is most probable to find the electron pair near the sulphur atom. In contrast, the cyclopentadienyl dipole moment is oriented towards the center of the ring due to resonance (see Figure 1). In this case, the asymmetric carbon has a total atomic charge of -0.099 . This asymmetry for the ring electric charge is measured by the LQSI-Coulomb (see Table 1). Additionally the thiophene dipole moment is smaller than the corresponding saturated tetrahydrothiophene. This is shown in Figure 5.

The dipole moment in the thiophene is influenced by resonance (see Figure 2). However, the effect of resonance is obviously not sufficient enough to exceed the effect of the electronegativity of sulphur. Thus, the negative pole is located at the heteroatom. However, due to the resonance, the dipole moments of thiophene are smaller than those of the corresponding saturated tetrahydrothiophene.

Induced molecular polarity index proposed for the thiophene molecule $\left(P_{\mathrm{kB}}\right)$ as function of the induced molecular polarity of cyclopentadienyl molecule $\left(P_{\mathrm{kA}}\right)$ (Table 2), taking into account the range of the Carbó index in accordance with the Schwartz integral inequality $[33,34]$.

Consider the following:

$$
\left[\int \rho_{\mathrm{A}}(r) \rho_{\mathrm{B}}(r) d r\right]^{2} \leq \int \rho_{\mathrm{A}}^{2}(r) d r \int \rho_{\mathrm{B}}^{2}(r) d r,
$$

where the similarity measure $\left(Z_{\mathrm{AB}}\right)$ is of (12).

In Table 2 the higher polarization values are to the thiophene. The molecular polarization (Figure 6) for the first quantum state (see Appendices A and B) is related by the proposed molecular descriptor (Table 2), expressing the molecular polarization of thiophene $\left(P_{\mathrm{kB}}\right)$ as function of molecular polarity of cyclopentadienyl $\left(P_{\mathrm{kA}}\right)$, for the groundstate 1 (Appendix A).

In Figure 6, we can see that this model suggests a new perspective on induced polar chemical bonding, due to the fact that the induced polarization process arises naturally 


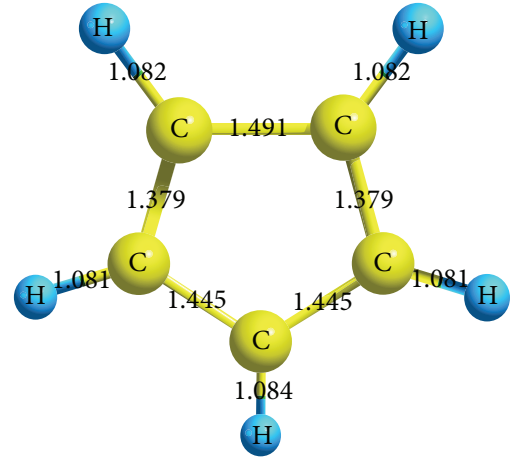

(a) Bond distance of cyclopentadienyl

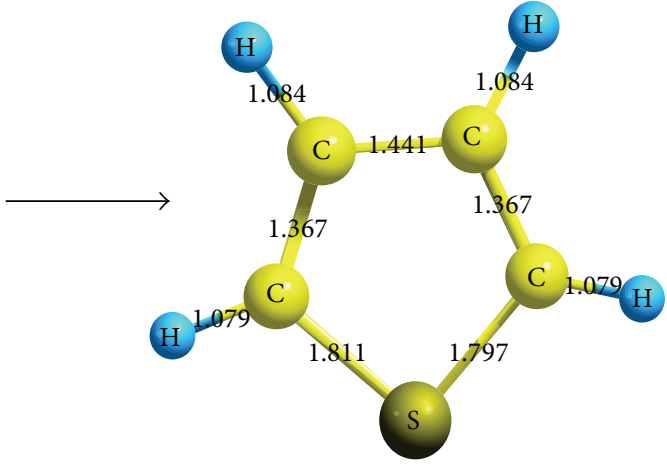

(b) Bond distance of thiophene

FIGURE 4: Comparison of the bond distances ( $\AA$ ) of (a) cyclopentadienyl and (b) thiophene, computed with ChemCraft 1.6 [32].

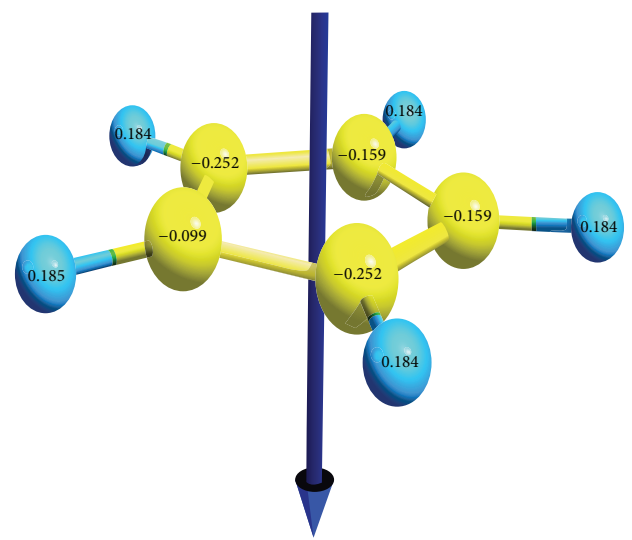

(a)

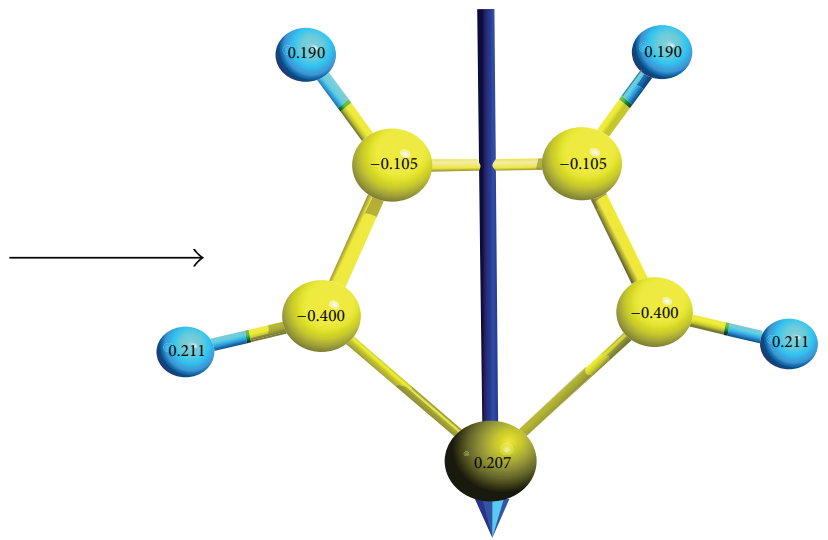

(b)

FIGURE 5: Dipole moment and total atomic charges (a) cyclopentadienyl molecules and (b) thiophene molecule.

as a result of elastic deformation of the cloud of electrons around atomic nuclei of the molecules A and B. Expressing the polarization induced by the molecule $\mathrm{A}$ in terms of the molecule $\mathrm{B}$, this new property is quantified by the proposed descriptor (molecular polarity):

$P_{1 \mathrm{~B}}$

$$
\begin{aligned}
& =\left(P_{1 \mathrm{~A}}\right)[\mathrm{SI}]=\left(\operatorname{Sp} \widehat{\rho}_{\mathrm{k}} \mathbf{D}\right)\left[R_{\mathrm{SC}}^{\text {local }}\right] \\
& =\left(\operatorname{Sp} \widehat{\rho}_{\mathrm{k}} \mathbf{D}\right) \\
& \times\left[\int\left(\int \frac{\rho_{\mathrm{S}}^{0}(r)}{\sum_{X} \rho_{X, \mathrm{~S}}^{0}(r)}\right)\left(\frac{\rho_{\mathrm{C}}^{0}(r)}{\sum_{Y} \rho_{Y, \mathrm{C}}^{0}(r)}\right) \rho_{\mathrm{S}}(r) \rho_{\mathrm{C}}(r) d r\right) \\
& \times\left(\int\left(\frac{\rho_{\mathrm{S}}^{0}(r)}{\sum_{X} \rho_{X, \mathrm{~S}}^{0}(r)}\right)^{2} \rho_{\mathrm{S}}(r) \rho_{\mathrm{S}}(r) d r\right]^{1 / 2} \\
& \left.\left.\quad \times\left[\int\left(\frac{\rho_{\mathrm{C}}^{0}(r)}{\sum_{Y} \rho_{Y, \mathrm{C}}^{0}(r)}\right)^{2} \times \rho_{\mathrm{C}}(r) \rho_{\mathrm{C}}(r) d r\right]^{1 / 2}\right)^{-1}\right] .
\end{aligned}
$$

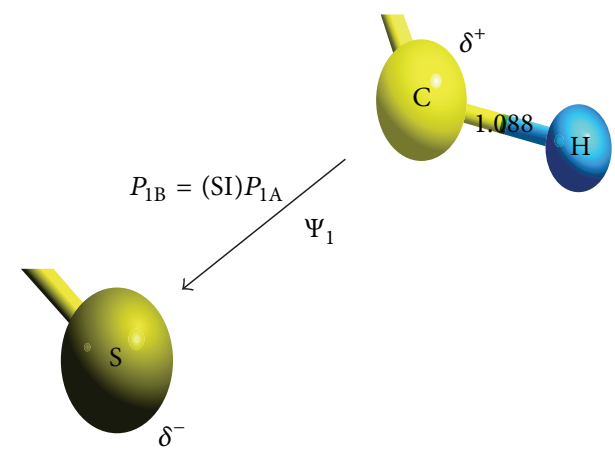

(SI): similarity index

FIGURE 6: Proposed molecular polarization descriptor in an external electric field in this study (18) for the state 1 of Appendix A; see Table 2.

On the other hand, this model includes interactions of the dipoles induced by the atoms when they are oriented in the electric dipole; in this sense the interactions between identical atoms in a molecule were identified as the induced polar bonds, considering that this model is not of point masses.

The importance of these new techniques lies in the finding of similar characteristics that allow us to correlate members 
TABLE 2: Induced molecular polarity index proposed for the thiophene and furan $\left(P_{\mathrm{kB}}\right)$ as function of the induced molecular polarity of cyclopentadienyl $\left(P_{\mathrm{kA}}\right)$, proposed in this study.

\begin{tabular}{|c|c|c|c|c|c|}
\hline \multicolumn{6}{|c|}{ Induced molecular polarity index for $P_{\mathrm{kB}}$} \\
\hline $2.994 P_{\mathrm{kA}}^{\mathrm{a}, \mathrm{b}}$ & $196.350 P_{\mathrm{kA}}^{\mathrm{a}, \mathrm{c}}$ & $0.972 P_{\mathrm{kA}}{ }^{\mathrm{d}}$ & $0.997 P_{\mathrm{kA}}{ }^{\mathrm{e}}$ & $0.410 P_{\mathrm{kA}}^{\mathrm{f}}$ & $0.966 P_{\mathrm{kA}}^{\mathrm{d}}$ \\
\hline $3.441 P_{\mathrm{kA}}$ & $211.863 P_{\mathrm{kA}}$ & $0.912 P_{\mathrm{kA}}$ & $0.991 P_{\mathrm{kA}}$ & $0.871 P_{\mathrm{kA}}$ & $2.009 P_{\mathrm{kA}}$ \\
\hline
\end{tabular}

${ }^{\mathrm{a}}$ Analytical method.

${ }^{\mathrm{b}}$ Interaction of overlap.

${ }^{\mathrm{c}}$ Interaction of coulomb.

${ }^{\mathrm{d}}$ Local similarity index of overlap (see Appendix C).

${ }^{\mathrm{e}}$ Local similarity index of coulomb (see Appendix C).

${ }^{\mathrm{f}}$ Euclidean distance of overlap.

${ }^{\mathrm{d}}$ Euclidean distance of coulomb.

$P_{\mathrm{kA}}:(7)$ with respect to the polarization states (Appendix B).

Note: $\mathrm{k}$ is the quantum state (Appendix A).

and physicochemical descriptors that quantify optical properties and quantum similarity; in that sense the ubiquitous nature of the similarity concept was well expressed by Rouvray, who stated that all issues of comparison and therefore of classification in the MQSI field can be related to the different dipole moments [35]. In addition, this model suggests a new perspective for the treatment of polar chemical bonding due to the fact that the polarization process arises naturally as a result of elastic deformation in the electronic cloud. These new techniques allow us to correlate molecular properties of systems, which differ only in one atom. We expect that in the future they can be applied to a wide range of compounds with major structural differences. Furthermore, this model can be considered as an approximation of a solvent model (i.e., a solvent can be modelled as a Polarizable Continuum Model (PCM), according to Tomasi group $[36,37])$ and can also be used towards the better understanding of the inductive effect in polar covalent bonds with external perturbation. In addition this study is complementary to the studies developed by Morales-Bayuelo et al. $[38,39]$ to understand the distortion of local orbitals by effects of an external electric field using the Hückel method, taking into account the charge transfer on the molecules by polarization effects according to Parr [40].

\section{Conclusions}

This study proposes a theoretical model of the local molecular polarization of thiopheneand furanunder the action of an electric field using Local Quantum Similarity Indexes (LQSI) based on the Hirshfeld partitioning in the (DFT) framework.

The hypothesis of neglecting the molecular polarization produced by neighboring atoms in the cyclopentadienyl is successful because the interatomic lengths are very similar on both sides of the ring, allowing us to focus our attention on the diatomic bond $(\mathrm{C}-\mathrm{C})$ of cyclopentadienyl and comparing it with the $(\mathrm{C}-\mathrm{S})$ bond of thiophene and $(\mathrm{C}-\mathrm{O})$ bond of furan. The most important descriptor of the local similarity was the coulomb index. It quantifies the differences between the dipole moments of the analyzed structures (see Figure 4 ). The method of alignment TGSA provides a straightforward procedure to solve the problem of relative molecular orientation, calculating molecular quantum similarity indexes. Additionally, this model can contribute to the interpretation of chemical bonds, molecular interactions, and distortion in the molecular polarization by action of an external electric field in the framework of the solvent effect theory [36-38, 41-45].

\section{Appendices}

\section{A. Quantum States Studied}

Quantum states for a transition $2 \mathrm{~s} \rightarrow 3 \mathrm{p}$ using (1):

$$
\begin{gathered}
\Psi_{1}=\Psi_{200} \bar{\Psi}_{200}, \quad \Psi_{2}=\Psi_{200} \bar{\Psi}_{31-1}, \quad \Psi_{3}=\Psi_{200} \bar{\Psi}_{310} \\
\Psi_{4}=\Psi_{200} \bar{\Psi}_{311}, \quad \Psi_{5}=\Psi_{31-1} \bar{\Psi}_{200}, \quad \Psi_{6}=\Psi_{310} \bar{\Psi}_{200} \\
\Psi_{7}=\Psi_{311} \bar{\Psi}_{200}, \quad \Psi_{8}=\Psi_{31-1} \bar{\Psi}_{31-1}, \\
\Psi_{9}=\frac{1}{\sqrt{2}}\left(\Psi_{31-1} \bar{\Psi}_{31-0}+\Psi_{310} \bar{\Psi}_{31-1}\right) \\
\Psi_{10}=\frac{1}{\sqrt{6}}\left(\Psi_{31-1} \bar{\Psi}_{311}+2 \Psi_{310} \bar{\Psi}_{310}+\Psi_{311} \bar{\Psi}_{31-1}\right) \\
\Psi_{11}=\frac{1}{\sqrt{2}}\left(\Psi_{310} \bar{\Psi}_{311}+\Psi_{311} \bar{\Psi}_{310}\right), \quad \Psi_{12}=\Psi_{311} \bar{\Psi}_{311} \\
\Psi_{13}=\frac{1}{\sqrt{2}}\left(\Psi_{310} \bar{\Psi}_{31-1}-\Psi_{31-1} \bar{\Psi}_{310}\right) \\
\Psi_{14}=\frac{1}{\sqrt{2}}\left(\Psi_{311} \bar{\Psi}_{31-1}-\Psi_{31-1} \bar{\Psi}_{311}\right) \\
\Psi_{15}=\frac{1}{\sqrt{2}}\left(\Psi_{311} \bar{\Psi}_{310}-\Psi_{310} \bar{\Psi}_{311}\right) \\
\left.\Psi_{311}-\Psi_{310} \bar{\Psi}_{310}+\Psi_{311} \bar{\Psi}_{31-1}\right)
\end{gathered}
$$




\section{B. Polarization States}

Polarization equations to the system using (7), for the molecule A (cyclopentadienyl):

$$
\begin{gathered}
P_{1 \mathrm{~A}}=4 X\left(E_{1} i+E_{2} j+E_{3} k\right), \\
P_{2 \mathrm{~A}}=\left(X E_{1}+Y E_{2}\right) i+\left(Y E_{1}+X E_{2}\right) j+2 X E_{3} k, \\
P_{3 \mathrm{~A}}=2 X\left(E_{1} i+E_{2} j\right), \\
P_{4 \mathrm{~A}}=\left(X E_{1}+Y E_{2}\right) i+\left(-Y E_{1}+X E_{2}\right) j+2 X E_{3} k, \\
\left\{P_{5 \mathrm{~A}}=P_{2 \mathrm{~A}}, P_{6 \mathrm{~A}}=P_{3 \mathrm{~A}}, P_{7 \mathrm{~A}}=P_{4 \mathrm{~A}}\right\}=\text { Degenerates states, } \\
P_{8 \mathrm{~A}}=-2\left[\left(X E_{1}-Y E_{2}\right) i+\left(-Y E_{1}+X E_{2}\right) j\right], \\
P_{9 \mathrm{~A}}=\left(-X E_{1}-Y E_{2}\right) i+\left(Y E_{1}-X E_{2}\right) j-2 X E_{3} k, \\
P_{10 \mathrm{~A}}=\frac{-2 X\left(E_{1} i+E_{2} j+4 E_{3} k\right)}{3}, \\
P_{11 \mathrm{~A}}=\left(-X E_{1}+Y E_{2}\right) i+\left(-Y E_{1}-X E_{2}\right) j-2 X E_{3} k, \\
P_{12 \mathrm{~A}}=-2\left[\left(X E_{1}-Y E_{2}\right) i+\left(Y E_{1}+X E_{2}\right) j\right], \\
P_{13 \mathrm{~A}}=\left(-X E_{1}+Y E_{2}\right) i+\left(Y E_{1}-X E_{2}\right) j-2 X E_{3} k, \\
P_{14 \mathrm{~A}}=-2 X\left(E_{1} i+E_{2} j\right), \\
P_{15 \mathrm{~A}}=\left(-X E_{1}+Y E_{2}\right) i+\left(-Y E_{1}-X E_{2}\right) j-2 X E_{3} k, \\
P_{16 \mathrm{~A}}=\frac{-4 X\left(E_{1} i+E_{2} j+E_{3} k\right)}{3},
\end{gathered}
$$

where

$$
\begin{gathered}
X=\frac{d^{2} \omega_{0}\left[\cos \omega_{0} t-\cos \omega t\right]}{3 \hbar\left[\omega^{2}-\omega_{0}^{2}\right]}, \\
Y=\frac{d^{2} \omega_{0}\left[\omega_{0} \sin \omega_{0} t-\omega \sin \omega t\right]}{3 \hbar\left[\omega^{2}-\omega_{0}^{2}\right]}, \\
d=-e \int_{0}^{\infty} R_{20} R_{31} r^{3} d r .
\end{gathered}
$$

$R_{20}, R_{31}$ represent the radial functions and $e$ represents the charge of a positron, with $\omega_{0}=\omega_{2}-\omega_{1}\left(\omega^{2} \neq \omega_{0}^{2}\right)$.

\section{On the Similarity Descriptors}

C.1. Overlap-Molecular Quantum Similarity (O-MQS). Using the formalism of the Dirac delta distribution $\Omega\left(r_{1}, r_{2}\right)=$ $\delta\left(r_{1}, r_{2}\right)$ [1], is possible to defined the so called overlap Molecular Quantum Similarity Measure and expresses the measure of the volume associated with the overlap of the two densities $\rho_{\mathrm{A}}$ and $\rho_{\mathrm{B}}$, and can be expressed mathematically as $[6-11,15,16]$

$$
\begin{aligned}
Z_{\mathrm{AB}}(\Omega) & =\iint \rho_{\mathrm{A}}\left(r_{1}\right) \delta\left(r_{1}-r_{2}\right) \rho_{\mathrm{B}}\left(r_{2}\right) d r_{1} d r_{2} \\
& =\int \rho_{\mathrm{A}}\left(r_{1}\right) \rho_{\mathrm{B}}\left(r_{2}\right) d r_{1} d r_{2} .
\end{aligned}
$$

Equation (A.1) provides information about the concentration of electrons in the molecule and indicates the degree of overlap between the compounds compared. In this sense this study calculated the overlap-MQSM for a description of the steric effects and determines its structural correlation.

C.2. Coulomb-Molecular Quantum Similarity (C-MQS). When the $\Omega$-operator in (15) is the coulomb operator $\Omega\left(r_{1}, r_{2}\right)=\left|r_{1}-r_{2}\right|^{-1}$, is obtained the coulomb-MQSM [46], representing the electronic coulomb repulsion energy between the two molecular densities $\rho_{\mathrm{A}}$ and $\rho_{\mathrm{B}}$, and can be expressed mathematically as

$$
Z_{\mathrm{AB}}(\Omega)=\iint \rho_{\mathrm{A}}\left(r_{1}\right) \frac{1}{\left|r_{1}-r_{2}\right|} \rho_{\mathrm{B}}\left(r_{2}\right) d r_{1} d r_{2} .
$$

The coulomb operator considers the molecular density function as electronic distribution in the space and calculates the coulomb repulsion between the electronic distributions (C.2). The similarity values are presented in matrix form.

\section{C.3. Similarity Matrix "Density Function Coordinates Matrix"} according to Carbó-Dorca [47]. The global set (QSM), which compares all the possible pair of quantum objects, is also expressed in matrix form, by means of the quantum similarity matrix (SM): $\mathbf{Z}=\{Z i j\}$, where $i, j \in(1, n)$.

The similarity matrix of dimension $(n \times n)$ can be defined as follows:

$$
\mathbf{Z}=\left\{Z_{i j}(\Omega) \mid \forall i, j \in M\right\} .
$$

With $\Omega$-operator, this matrix can be also considered as a hypervector formed by a set of column vectors: $\mathbf{Z}=$ $\left\{Z_{1}, Z_{2}, \ldots, Z_{n}\right\}$, where each column (or row) vector is formed by the collection of all the similarity indexes related to the quantum objects that are all the MQS-indexes between the ath molecule and each element of the set, including the self-similarity $\left(Z_{n n}\right)$. Consequently every $\left(Z_{a}\right)$ is interpreted as a discrete $N$-dimensional representation of the $a$ th structure:

$$
\mathbf{Z}=\begin{gathered}
1 \\
2 \\
3 \\
\vdots \\
j \\
\quad \vdots \\
n \\
n
\end{gathered}\left[\begin{array}{ccccccc}
1 & 2 & 3 & \cdots & i & \cdots & n \\
Z_{11} & & & & & & \\
Z_{12} & Z_{22} & & & & & \\
Z_{13} & Z_{23} & Z_{33} & & & & \\
& \vdots & & \ddots & & & \\
Z_{1 j} & Z_{2 j} & \vdots & & Z_{j i} & & \\
\vdots & & \vdots & & \vdots & \ddots & \\
Z_{1 n} & Z_{2 n} & Z_{3 n} & \cdots & Z_{n i} & \cdots & Z_{n n}
\end{array}\right] .
$$

Generic quantum similarity matrix, $\mathbf{Z}$ for $(n)$ quantum objects, with the $n(n+1) / 2$ pairwise calculations for the lower triangle. The self-similarity is present when $j=i$, using the recent definition proposed by Carbó-Dorca for this matrix "density function coordinates matrix" [47].

\section{Conflict of Interests}

The authors declare that there is no conflict of interests regarding the publication of this paper. 


\section{Acknowledgments}

Alejandro Morales-Bayuelo thanks the Universidad Nacional Andres Bello (Santiago, Chile) for a Ph.D. fellowship (CONICYT (63100003)). Ricardo Vivas-Reyes is indebted to the Universidad de Cartagena (Cartagena de Indias, Colombia), for continuous support to his group. The authors wish to thank Professor Ramon Carbó-Dorca (Girona, Spain) for numerous constructive remarks on an early version of this text.

\section{References}

[1] F. L. Hirshfeld, "Bonded-atom fragments for describing molecular charge densities," Theoretica Chimica Acta, vol. 44, pp. 129138, 1997.

[2] R. Carbó-Dorca, L. Amat, E. Besalú, X. Gironés, and D. Robert, "Quantum mechanical origin of QSAR: theory and applications," Journal of Molecular Structure, vol. 504, no. 1-3, pp. 181228, 2000.

[3] N. Ferro, P. Bultinck, A. Gallegos, H.-J. Jacobsen, R. CarbóDorca, and T. Reinard, "Unrevealed structural requirements for auxin-like molecules by theoretical and experimental evidences," Phytochemistry, no. 68, pp. 237-250, 2007.

[4] R. Carbó-Dorca, E. Besalfi, L. Amat, and X. Fradera, "On quantum molecular similarity measures (QMSM) and indices (QMSI)," Journal of Mathematical Chemistry, vol. 19, pp. 47-56, 1996.

[5] C. Cohen-Tannoudji, "Upravlenie atomami s pomoshch'yu fotonov," Uspekhi Fizicheskikh Nauk, vol. 169, no. 3, p. 292, 1999.

[6] R. Carbó, M. Arnau, and L. Leyda, "How similar is a molecule to another? An electron density measure of similarity between two molecular structures," International Journal of Quantum Chemistry, vol. 17, pp. 1185-1189, 1980.

[7] X. Gironés and R. Carbó-Dorca, "Modelling toxicity using molecular quantum similarity measures," QSAR \& Combinatorial Science, vol. 25, no. 7, pp. 579-589, 2006.

[8] R. Carbó-Dorca, E. Besalú, and L. D. Mercado, "Communications on quantum similarity, part 3: a geometric-quantum similarity molecular superposition algorithm," Journal of Computational Chemistry, vol. 32, no. 4, pp. 582-599, 2010.

[9] R. Carbó-Dorca and X. Gironés, "Foundation of quantum similarity measures and their relationship to QSPR: density function structure, approximations, and application examples," International Journal of Quantum Chemistry, vol. 101, no. 1, pp. 8-20, 2005.

[10] R. Carbó-Dorca and E. Besalú, "Communications on quantum similarity (2): a geometric discussion on holographic electron density theorem and confined quantum similarity measures 2010," Journal of Computational Chemistry, vol. 31, no. 13, pp. 2452-2462.

[11] M. M. Deza and E. Deza, Encyclopedia of Distances, Springer, Berlin, Germany, 2009.

[12] L. Amat and R. Carbó-Dorca, "Use of promolecular ASA density functions as a general algorithm to obtain starting MO in SCF calculations," International Journal of Quantum Chemistry, vol. 87, pp. 59-67, 2002.

[13] I. I. Khvalchenko, "Polarization of two identical atoms in an electric field," Russian Physics Journal, vol. 48, no. 7, pp. 729732, 2005.
[14] E. Wigner, Group Theory and Its Application to the Quantum Mechanics of Atomic Spectra, Academic Press, New York, NY, USA, 1959.

[15] S. K. Berberian, Introduction to Hilbert Space, Oxford University Press, New York, NY, USA, 1961.

[16] R. Vivas-Reyes, A. Arias, J. Vandenbussche, C. van Alsenoy, and P. Bultinck, "Quantum similarity of isosteres coordinate versus momentum space and influence of alignment," Journal of Molecular Structure: THEOCHEM, vol. 943, no. 1-3, pp. 183188, 2010.

[17] P. Bultinck, X. Girones, and R. Carbó-Dorca, "Molecular quantum similarity: theory and applications," Reviews in Computational Chemistry, vol. 21, pp. 127-207, 2005.

[18] P. Bultinck and R. Carbó-Dorca, "A mathematical discussion on density and shape functions," Journal of Mathematical Chemistry, vol. 36, no. 2, pp. 191-200, 2004.

[19] P. Bultinck, W. Langenaeker, P. Lahorte et al., "The electronegativity equalization method II: applicability of different atomic charge schemes," The Journal of Physical Chemistry A, vol. 106, no. 347901, p. 7895, 2002, 10. 1021/jp020547v.

[20] F. De Proft, C. van Alsenoy, A. Peeters, W. Langenaeker, and P. Geerlings, "Atomic charges, dipole moments, and Fukui functions using the Hirshfeld partitioning of the electron density," Journal of Computational Chemistry, vol. 23, no. 12, pp. 1198-1209, 2002.

[21] F. De Proft, R. Vivas-Reyes, A. Peeters, C. van Alsenoy, and P. Geerlings, "Hirshfeld partitioning of the electron density: atomic dipoles and their relation with functional group properties," Journal of Computational Chemistry, vol. 24, pp. 463-470, 2003.

[22] G. Boon, C. van Alsenoy, F. De Proft, P. Bultinck, and P. Geerlings, "Molecular quantum similarity of enantiomers of amino acids: a case study," Journal of Molecular Structure: THEOCHEM, vol. 727, no. 1-3, pp. 49-66, 2005.

[23] X. Girones, D. Robert, and R. Carbó-Dorca, “TGSA: a molecular superposition program based on topo-geometrical considerations," Journal of Computational Chemistry, vol. 22, no. 2, pp. 255-263, 2001

[24] E. E. Hodgkin and W. G. Richards, "Molecular similarity based on electrostatic potential and electric field," International Journal of Quantum Chemistry, vol. 32, 14, pp. 105-110, 1987.

[25] A. Morales-Bayuelo, H. Ayazo, and R. Vivas-Reyes, "Threedimensional quantitative structure-activity relationship CoMSIA/CoMFA and Leapfrog studies on novel series of bicyclo [4.1.0] heptanes derivatives as melanin-concentrating hormone receptor R1 antagonists," European Journal of Medicinal Chemistry, pp. 4509-4522, 2010.

[26] M. Ahumedo, A. Díaz, and R. Vivas-Reyes, "Theoretical and structural analysis of the active site of the transcriptional regulators LasR and TraR, using molecular docking methodology for identifying potential analogues of acyl homoserine lactones (AHLs) with anti-quorum sensing activity," European Journal of Medicinal Chemistry, vol. 45, no. 2, pp. 608-615, 2010.

[27] L. Chen, "Substructure and maximal common substructure searching," in ComPutational Medicinal Chemistry For Drug Discovery, P. Bultinck, H. De Winter, W. Langenaeker, and J. P. Tollenaere, Eds., Marcel Dekker, New York, NY, USA, 2003.

[28] A. D. Becke, "Density-functional thermochemistry. III. The role of exact exchange," The Journal of Chemical Physics, vol. 98, article 5648, 1993.

[29] C. Lee, W. Yang, and R. G. Parr, "Development of the collesalvetti correlation-energy formula into a functional of the 
electron density," Physical Review B, vol. 37, no. 5, pp. 785-789, 1988.

[30] G. Chalasinski and M. Szczesniak, "Origins of structure and energetics of van der Waals clusters from ab initio calculations," Chemical Reviews, vol. 94, no. 7, pp. 1723-1765, 1994.

[31] M. J. Frisch, G. W. Trucks, H. B. Schlegel et al., GAUSSIAN 09, Revision C. 01, Gaussian, Wallingford, Conn, USA, 2010.

[32] ChemCraft 1. 6, is a Windows-based graphical program for working with quantum chemistry calculations. It is an affordable and convenient tool for visualization of chemical data and preparing new jobs for calculation. Programming: G. A. Zhurko, site design, additional support: D. A. Zhurko, Web programming: A. Romanov, 2011, http://www.chemcraftprog.com/.

[33] V. Neumann, Mathematical Foundations of Quantum Mechanics, , Princeton University Press, Princeton, NJ, USA, 1955.

[34] I. M. Vinogradov, Encyclopaedia of Mathematics, Kluwer Academic Publishers, Dordrecht, The Netherlands, 1989.

[35] D. H. Rouvray, "Similarity in chemistry-past, present and future," in Molecular Similarity I, vol. 173 of Topics in Current Chemistry, pp. 1-30, 1995.

[36] J. Tomasi, B. Mennucci, and R. Cammi, "Quantum mechanical continuum solvation models," Chemical Reviews, vol. 105, pp. 2999-3093, 2005.

[37] B. Mennucci, Tomasi, R. Cammi et al., "Polarizable continuum model (PCM) calculations of solvent effects on optical rotations of chiral molecules," The Journal of Physical Chemistry A, vol. 106, no. 25, pp. 6102-6113, 2002.

[38] A. Morales-Bayuelo and R. Vivas-Reyes, “Theoretical model for the polarization molecular and Hückel treatment of PhosphoCyclopentadiene in an external electric field: hirschfeld study," Journal of Mathematical Chemistry, vol. 51, no. 7, pp. 1835-1852, 2013.

[39] A. Morales-Bayuelo, J. Torres, and R. Vivas-Reyes, "Hückel treatment of pyrrole and pentalene as a function of cyclopentadienyl using local quantum similarity index (LQSI) and the topo-geometrical superposition approach (TGSA)," Journal of Theoretical and Computational Chemistry, vol. 11, no. 1, article 223, 2012.

[40] R. G. Parr, "Remarks on the concept of an atom in a molecule and on charge transfer between atoms on molecule formation," International Journal of Quantum Chemistry, vol. 26, no. 5, pp. 687-692, 1984.

[41] T. N. Truong and E. V. Stefanovich, "A new method for incorporating solvent effect into the classical, ab initio molecular orbital and density functional theory frameworks for arbitrary shape cavity," Chemical Physics Letters, vol. 240, no. 4, pp. 253-260, 1995.

[42] E. G. McRae, "Theory of solvent effects on molecular electronic spectra. Frequency shifts," The Journal of Physical Chemistry A, vol. 61, no. 5, pp. 562-572, 1957.

[43] M. Cossi and V. Barone, "Time-dependent density functional theory for molecules in liquid solutions," Journal of Chemical Physics, vol. 115, no. 10, pp. 4708-4717, 2001.

[44] J. Yu and M. C. Zerner, "Solvent effect on the first hyperpolarizabilities of conjugated organic molecules," Journal of Chemical Physics, no. 100, article 7487, 1994.

[45] M. Cossi and V. Barone, "Solvent effect on vertical electronic transitions by the polarizable continuum model," The Journal of Chemical Physics, vol. 112, pp. 2427-2435, 2000.

[46] R. Carbó-Dorca and L. Domingo, "LCAO-MO similarity measures and taxonomy," International Journal of Quantum Chemistry, vol. 32, no. 4, pp. 517-545, 1987.
[47] R. Carbó-Dorca, "On density function coordinate matrix," Journal of Mathematical Chemistry, vol. 51, no. 7, pp. 1681-1683, 2013. 

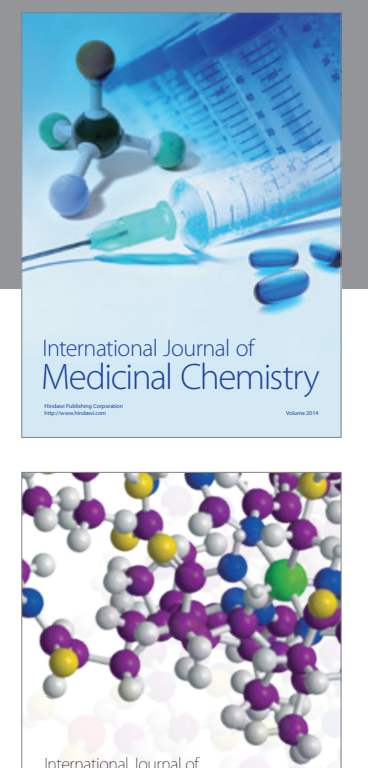

\section{Carbohydrate} Chemistry

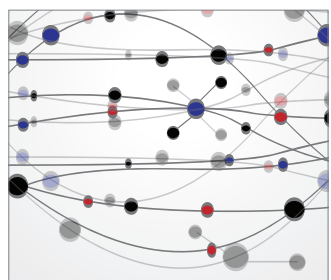

The Scientific World Journal
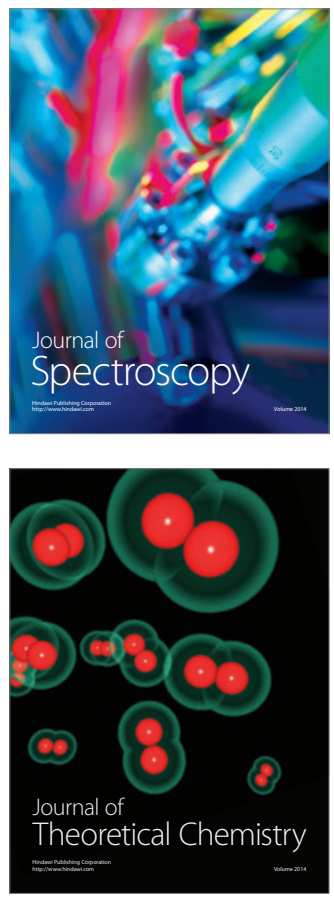
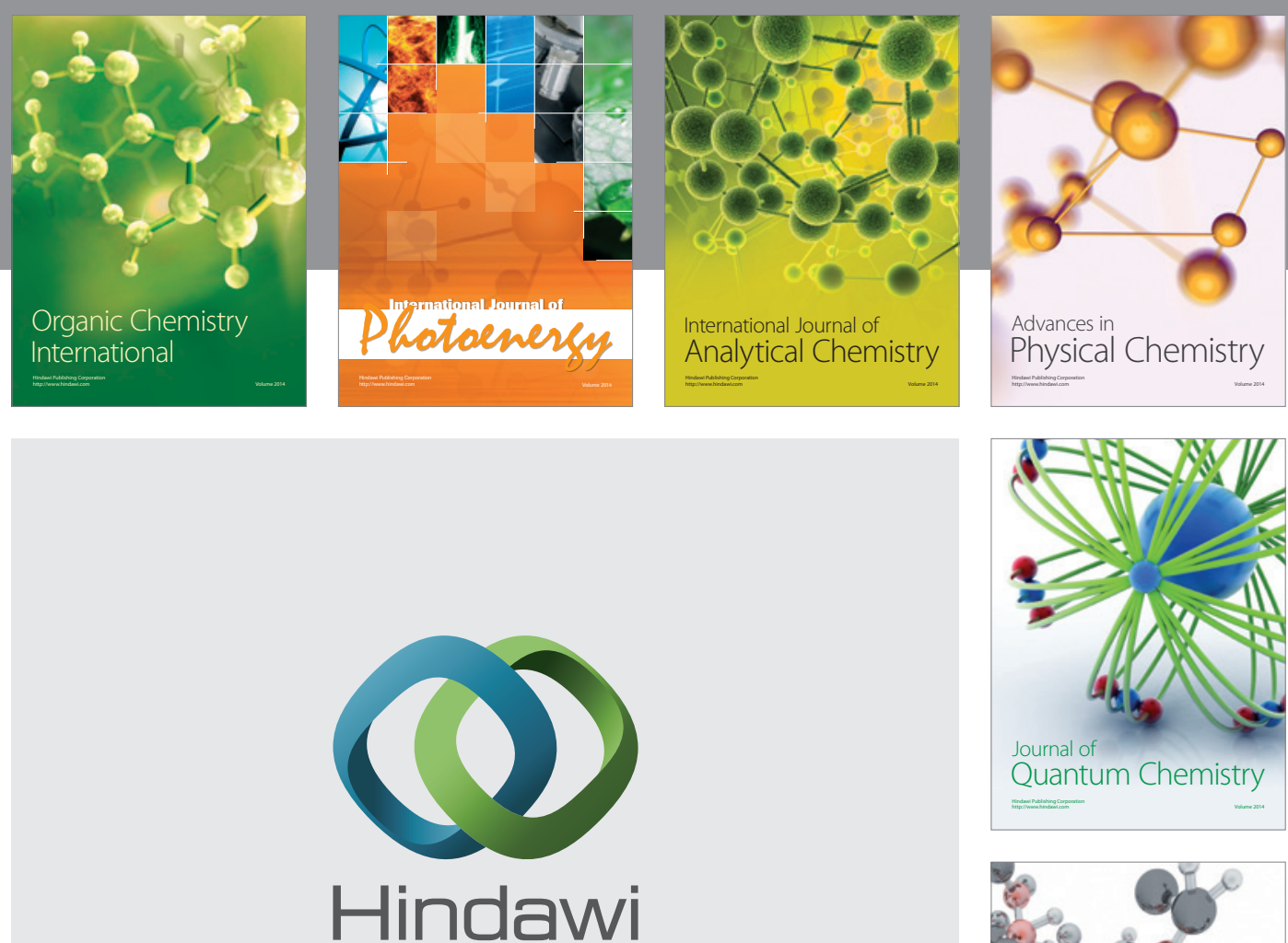

Submit your manuscripts at

http://www.hindawi.com

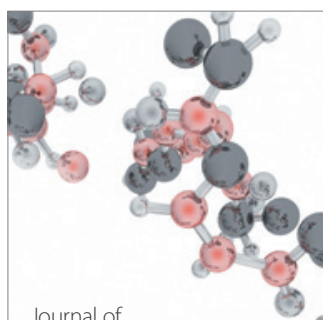

Analytical Methods

in Chemistry

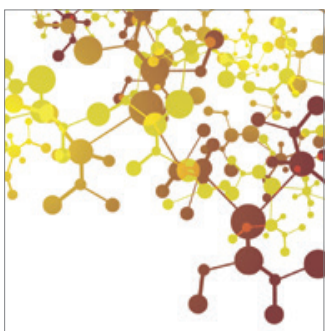

Journal of

Applied Chemistry

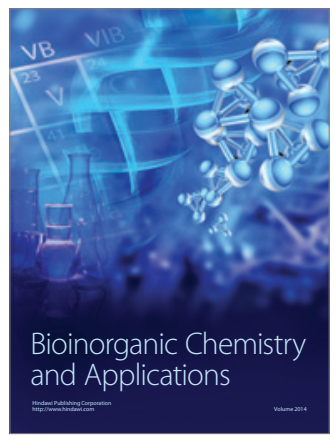

Inorganic Chemistry
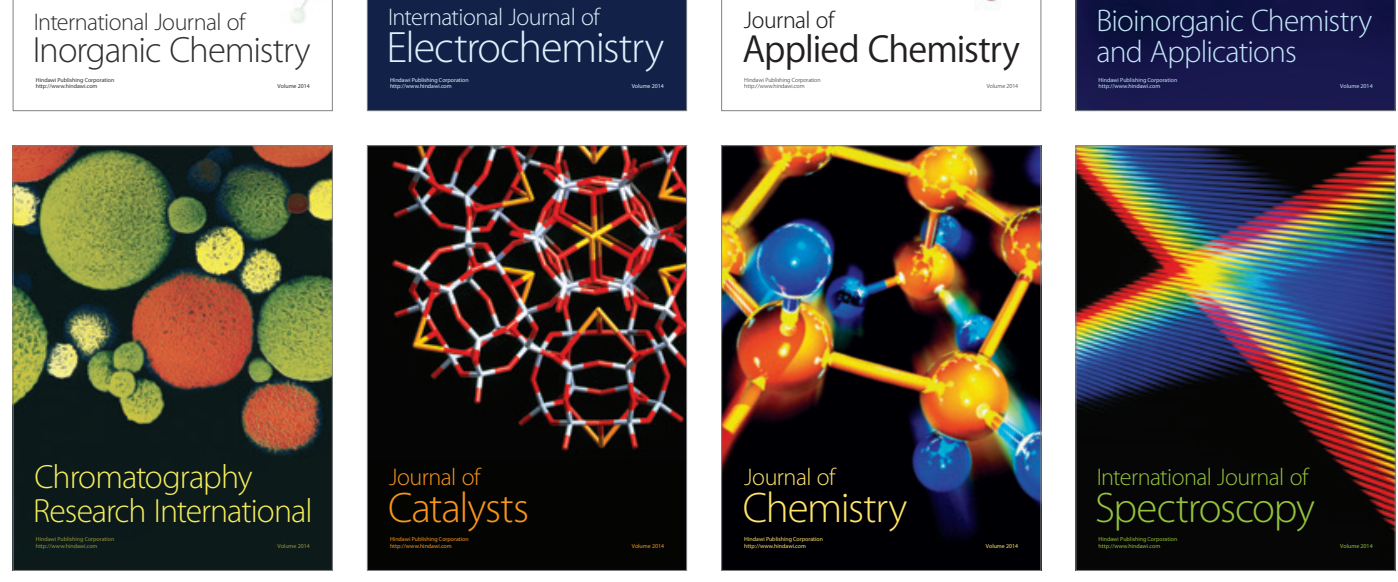